Journal of Animal and Veterinary Advances 11 (15): 2630-2640, 2012

ISSN: $1680-5593$

(C) Medwell Journals, 2012

\title{
Geometrical Study on FMDV Genome Based on Z-Curve
}

\author{
Hua Tang, Xin-Sheng Liu, Yu-Zhen Fang, Li Pan, Zong-Wang Zhang, Peng Zhou, \\ Jian-Liang Lv, Shou-Tian Jiang, Yong-Lu Wang and Yong-Guang Zhang \\ State Key Laboratory of Veterinary Etiological Biology, \\ National Foot and Mouth Disease Reference Laboratory, Lanzhou Veterinary Research Institute, \\ Chinese Academy of Agricultural Sciences, 730046 Lanzhou, China
}

\begin{abstract}
The Z-curve is a geometrical tool for visualizing and comparing genomes. Since, the curve contains the information carried by the given sequence, DNA sequences could be analyzed systematically. In this study, the Foot and Mouth Disease Virus (FMDV) was analyzed by the Z-curve Method. This research concludes that all serotypes FMDV have a close consanguinity but each has its own characteristic by contrast, the three South African serotypes have a much closer affinity. FMDV have three A $+\mathrm{T}$-rich regions and two $\mathrm{G}+\mathrm{C}$-rich regions. The most variable regions of FMDV lie in protein coding areas. And the non-coding region is very conservative. O serotype FMDV is most variable compared with A and Asial serotype. Those O serotype FMDV in buffalo and goat have a mutation trend. Compared with host, geographic location have a grater influence on FMDV mutation. The A serotype FMDV strains occurred in India after 2002 are more stable and have a closer phylogenetic relationships compared with the Asial strains which were prevalent before 2002 . In general, Z-curve Method shows a widely application prospect in the areas of life sciences. Such as gene sequence, molecular epidemiology, homology, evolution, phylogenetic relationships and genetic diversity analysis.
\end{abstract}

Key words: Z-curve, geometrical study, FMDV, mutation, homology analysis, China

\section{INTRODUCTION}

The increasing genome sequences urge us to exploit some methods to study these sequences. Currently, geometric approach is widely used to analysis DNA sequences and amino acid sequences. The merits of graphical research of these sequences are: intuitive, visual and vivid. Graphical models have been frequently used to describe complex interaction patterns and dependent structures among genes and other phenotypes (Chu et al., 2009). Li et al. (2010) introduced a cluster-free algorithm based on a graph-regularized version of Partial Least Squares (PLS) regression to learn sequence patterns represented by graphs of k-mers or graph-mers that predict gene expression trajectories. Giacomo et al. (2007) applied a graph-based approach to identify cancer signaling pathways from published gene expression signatures.

Z-curve is a 3-dimensional graph which is unique to the given the DNA sequence. That is to say, this graph contains the information carried by the given sequence. Since it was first proposed, the Z-curve has been extensively applied to life science research such as sequence segmentation (Zhang and Zhang, 2003a; Wen and Zhang, 2003), horizontal gene transfer detection (Zhang and Zhang, 2003b), isochoric domain inference (Zhang and Zhang, 2003a, b; Wen and Zhang, 2003) and sequence analysis, for instance, nucleotide distribution analysis (Ou et al., 2003), replication origins Identification (Zhang and Zhang, 2005), protein coding genes recognition (Zhang and Wang, 2000), separate base usages of genes revelation (Guo and $\mathrm{Yu}, 2007$ ) and so on. People have compared Z-curve Version 1.0 and Glimmer version 2.02 which was developed by the American Institute of Genomic Research. The results indicated that $\mathrm{Z}$-curve version 1.0 has a much lower false positive rate than Glimmer 2.02, especially for high $\mathrm{G}+\mathrm{C}$ content bacterial and archaealgenomes. What's more Z-curve 1.0 has more accurate gene start prediction, lower additional prediction rate and higher accuracy for the prediction of horizontally transferred genes (Guo et al., 2003). Because of its outstanding performance, Z-curve version 1.0 attracted widely attention in the filed of international bioinformatics and genomics. Foot and Mouth Disease (FMD) is an acute, highly contagious

Corresponding Author: Yong-Lu Wang, State Key Laboratory of Veterinary Etiological Biology,

National Foot and Mouth Disease Reference Laboratory, Lanzhou Veterinary Research Institute, Chinese Academy of Agricultural Sciences, 730046 Lanzhou, China 
disease of cloven-hoofed animals and is endemic in many regions in Africa, Asia and South America (Alexandersen and Mowat, 2005; Davies, 2002; Thompson et al., 2002; Knowles and Samuel, 2003; Cottam et al., 2008; Schumann et al., 2008). It is caused by the Foot and Mouth Disease Virus (FMDV), a member of the genus Aphthovirus of the family Picornaviridae which is highly transmissible and can causes high morbidity outbreaks with moderate to low mortality in most cases. There are seven serotypes of FMDV (A, O, C, Asial, SAT1, SAT2 and SAT3) with multiple subtypes within each serotype (Paprocka, 2006). The FMDV is a positive sense, single-stranded RNA virus. The genome is about 8,000 nucleotides (nt) in length. The harmfulness of FMD is conspicuous (Sumption et al., 2008; Gibbs, 2003; Sobrino et al., 2001) and there is no effective way to control this disaster (Mort et al., 2005). FMD is perhaps the most important animal disease limiting trade of animals and animal products (Rufael et al., 2008; Barasa et al., 2008; Perry and Rich, 2007). No effective drugs are currently available to cure this disease. It is unlikely that a single vaccine approach will solve the many short comings of current vaccines (Rodriguez and Grubman, 2009). Gaining insight into the information carried by the genomes of FMDV would be helpful to discover drugs and develop vaccines.

\section{MATERIALS AND METHODS}

The FMDV genomes and annotated information were downloaded at the web (site http://www.ncbi.nlm.nih. gov/). The Z-curve coordinates compute software (Zplotter) was downloaded at the web site (http://tubic. tju.edu.cn/zcurve/). Users are recommended to use Z-curve plotter Java applet version which can be available at the website (http:/ttubic.tju.edu.cn/zcurve/). However, for those who experience difficulty in using Java applet or want to compute Z-curve coordinates locally, this local version of $Z$ plotter can be used.

The Z-curve: The Z-curve is a 3-dimensional space curve which consists of a series of nodes Pn where $\mathrm{n}=0,1,2, \ldots$, $\mathrm{N}$ whose coordinates are calculated by Eq. 1 (Damien and Fares, 2006):

$$
\begin{aligned}
& \left\{\begin{array}{l}
\mathrm{x}_{\mathrm{n}}=(\mathrm{An}+\mathrm{Gn})-(\mathrm{Cn}+\mathrm{Tn}) \equiv \mathrm{Rn}-\mathrm{Yn} \\
\mathrm{y}_{\mathrm{n}}=(\mathrm{An}+\mathrm{Cn})-(\mathrm{Gn}+\mathrm{Tn}) \equiv \mathrm{Mn}-\mathrm{Kn} \\
\mathrm{z}_{\mathrm{n}}=(\mathrm{An}+\mathrm{Tn})-(\mathrm{Cn}+\mathrm{Gn}) \equiv \mathrm{Wn}-\mathrm{Sn}
\end{array}\right. \\
& \mathrm{n}=0,1,2, \ldots, N, \mathrm{x}_{\mathrm{n}}, \mathrm{y}_{\mathrm{n}}, \mathrm{z}_{\mathrm{n}} \in[\mathrm{N}, \mathrm{N}]
\end{aligned}
$$

where, $A_{0}=C_{0}=G_{0}=T_{0}=0$ and $x_{0}=y_{0}=z_{0}=0$. In the equation, $\mathrm{R}, \mathrm{Y}, \mathrm{M}, \mathrm{K}, \mathrm{W}$ and $\mathrm{S}$ represent the bases of purine, pyrimidine, amino, keto, weak hydrogen bonds and strong hydrogen bonds, respectively. Usually for an AT-rich genome, $z_{n}$ is approximately a monotonously increasing linear function of $\mathrm{n}$ whereas for a GC-rich genome, $\mathrm{z}_{\mathrm{n}}$ is approximately a monotonously decreasing linear function of $\mathrm{n}$. In both cases, it is convenient to fit the curve of $\mathrm{z}_{\mathrm{n}} \sim \mathrm{n}$ which is defined in Eq. 2 (Zhang and Zhang, 2003a, b):

$$
\mathrm{z}=\mathrm{kn}
$$

However in practice, the resolution ratio of $\mathrm{z}=\mathrm{kn}$ curve is very low, so in order to view the relevant graph more intuitively, the designer also introduce a $z_{n}{ }^{\prime} \sim n$ curve (simply as Z-curve), it is defined in Eq. 3 (Zhang and Zhang, 2003a, b):

$$
\mathrm{z}_{\mathrm{n}}{ }^{\prime}=\mathrm{z}_{\mathrm{n}}-\mathrm{kn}
$$

where, let $\overline{\mathrm{G}+\mathrm{C}}$ denote the average $\mathrm{G}+\mathrm{C}$ content within a region $\Delta \mathrm{n}$ in a sequence, researchers deduce Eq. 4 (Zhang and Zhang, 2003a, b):

$$
\overline{\mathrm{G}+\mathrm{C}}=\frac{1}{2}\left(1-\mathrm{k}-\frac{\Delta \mathrm{z}_{\mathrm{n}}{ }^{\prime}}{\Delta \mathrm{n}}\right) \equiv \frac{1}{2}\left(1-\mathrm{k}-\mathrm{k}^{\prime}\right)
$$

\section{RESULTS AND DISCUSSION}

Study on the seven FMDV serotypes: In order to study the whole serotypes FMDV, corresponding FMDV (Table 1) complete genome sequences are analyzed by Z-curve. The results indicate that all serotypes FMDV have a close consanguinity but each has its own

\begin{tabular}{|c|c|c|c|}
\hline Mark & Strain annotated in NCBI & Accession no. & The global distribution of ACGT base \\
\hline a\13 & a5allier iso45 & AY593780.1 & A:2043 C:2315 G:2118 T:1723 GC content:54.60\% \\
\hline $0 \backslash 13$ & o5india iso34 & AY593828.1 & A:2007 C:2301 G:2110 T:1739 GC content:54.08\% \\
\hline$c \backslash 13$ & C1 Oberbay ern c1 ober iso 88 & AY593805.1 & A:2031 C:2313 G:2073 T:1725 GC content:53.87\% \\
\hline Asia1 $\backslash 10$ & YNBS/58 & AY390432.1 & A:2013 C:2263 G:2105 T:1782 GC content:53.51\% \\
\hline Sat1 12 & $\mathrm{SAT} 1 / 5 \mathrm{sa} / 61$ isol3 & AY593842.1 & A:2072 C:2366 G:2012 T:1726 GC content:53.55\% \\
\hline Sat $2 \backslash 8$ & SAT2-1rhod/48 & AY593847.1 & A:2056 C:2321 G:2017 T:1725 GC content:53.43\% \\
\hline Sat312 & SAT3-3kenya $11 / 60$ & AY593852.1 & A:2046 C:2316 G:2035 T:1767 GC content:53.22\% \\
\hline
\end{tabular}
characteristic, by contrast, the three South Africa FMDV have a much closer affinity (Fig. 1a, b). The most variable regions of FMDV lie in protein coding areas and the

Table 1: The mark and name and the NCBI accession number as well as the global ACGT base distribution of the seven serotypes FMDV strains mapped 

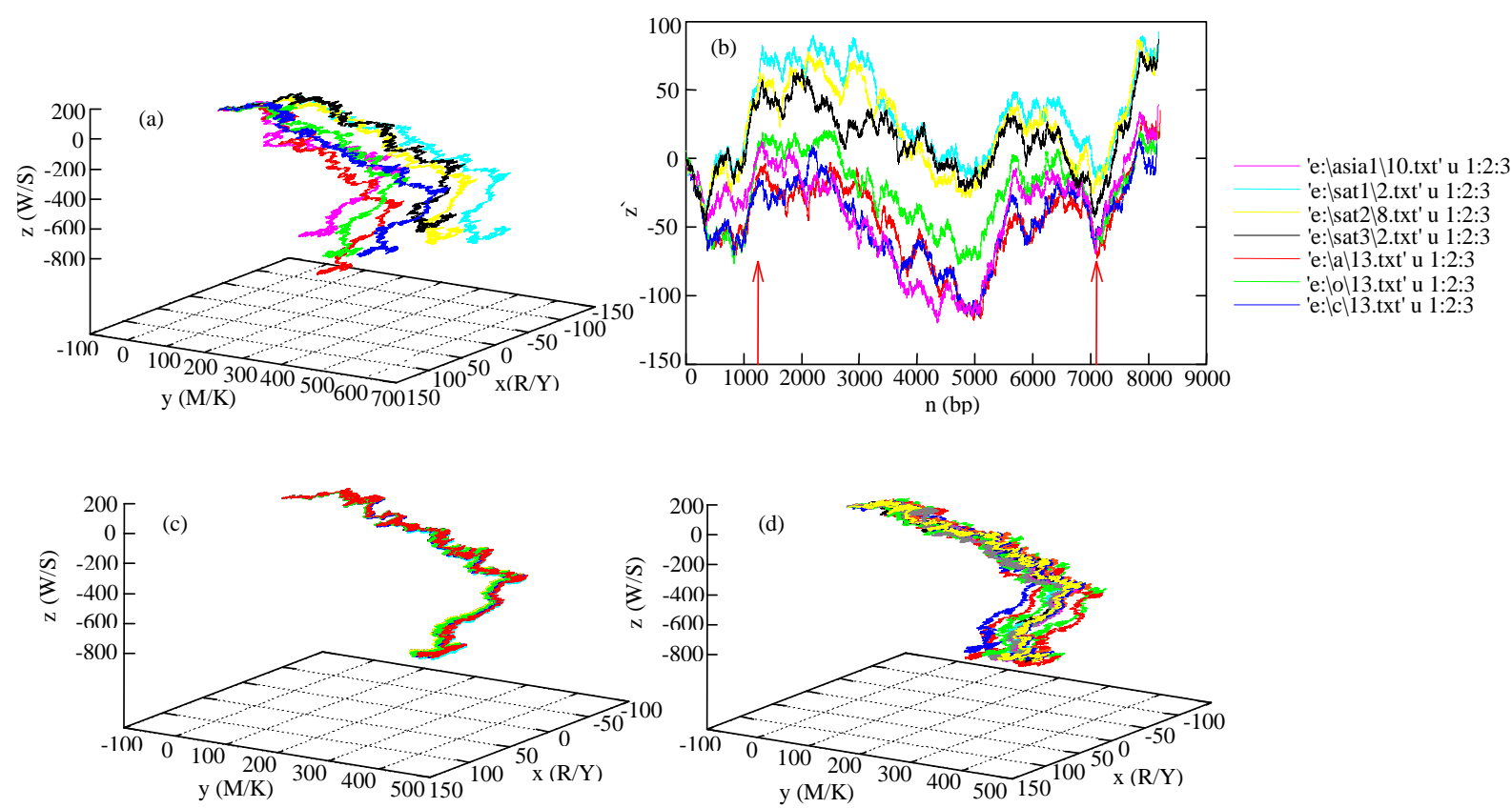

Fig. 1: a-d) Z-curves and the 3-dimensional curves of 7 serotypes FMDV strains

non-coding region is very conservative (Fig. 1b) and FMDV have three $\mathrm{A}+\mathrm{T}$-rich and two $\mathrm{G}+\mathrm{C}$-rich regions. And the $\mathrm{G}+\mathrm{C}$-rich regions are immediately followed by $\mathrm{A}+\mathrm{T}$-rich regions (Fig. 1b).

The mutation research of $O$ serotype FMDV based on host and geographic location: Here, 12 FMDV strains isolated in United Kingdom which have different hosts and another 12 strains isolated in different regions which have an identical host (Table 2 and 3) are analyzed to study the mutation of FMDV based on host and geographic location. And the former show a much closer consanguinity than the latter. Results also show that the impact of geographic location on FMDV mutation is greater than that of host (Fig. 1c,d). An up jump in the $\mathrm{Z}$-curve indicates a decrease of $\mathrm{G}+\mathrm{C}$ content whereas a drop indicates an increase of $\mathrm{G}+\mathrm{C}$ content. Any minimum point in the $Z$-curve indicates an abrupt change from $\mathrm{G}+\mathrm{C}$-rich region to $\mathrm{G}+\mathrm{C}$-poor region. The most different region (between the two arrows) means a most variable region of FMDV. The 3-dimensional curves of which are more similar and overlap better have closer consanguinity. Figure 1a, b indicates that the three South Africa serotypes have a much closer affinity and the most variable regions of FMDV lie in protein coding areas; Fig. 1c shows the 3-dimensional curves of 12 strains in United Kingdom which have different hosts and Fig. 1d shows the 3-dimensional curves of 12 strains in Asia and Europe which have the same host cattle. Figure $1 \mathrm{a}, \mathrm{b}$ shows that compared with host, geographic location have a grater influence on FMDV mutation.
The mutation research of $O$ serotype FMDV based on host: Researchers have studied the mutation of $O$ serotype FMDV based on their hosts. The $O$ serotype FMDV (Table 2 and 3) which have host annotated and complete genome available in NCBI are analyzed by $Z$-curve. The results indicate that $O$ serotype FMDV is most stable in sheep and most variable in swine (Fig. 2a-c). Israel 07-6378 (goat) is very similar to $\mathrm{PAK} / 45 / 2008$ (buffalo) while they are obviously different from others in the foreside. So, researchers speculate that $O$ serotype FMDV has a mutation trend in goat and buffalo. And researchers guess that there is a certain relationship between their occurrence when taking the collection time and isolation region into consideration (Fig. 2b-d). Figure 2b shows the 3-dimensional curves of $O$ serotype FMDV isolated in sheep. Fig. 2c is 3-dimensional curve of $O$ serotype FMDV isolated in bovine and Fig. 2 shows the 3-dimensional curves of Israel 07-6378 (goat) and PAK/45/2008 (buffalo). These illustrate that the $O$ serotype is most stable in sheep and most variable in swine. In addition, the $O$ serotype has a mutation trend in goat and buffalo. And may be, there is a certain relationship between the occurrence of Israel 07-6378 (goat) and PAK/45/2008 (buffalo).

The mutability research of Asia1, A, O, serotypes: Corresponding Asial, A, O, serotypes (Table 2, 4 and 5), complete genomes have been conducted Z-curve analyses, arming to study the mutability of them. According to the results, the O serotype FMDV is most variable compared with Asial and A serotype (Fig. 3a-c). 
Table 2: The Z-curve plot marks, names, accession numbers, hosts as well as isolation regions and collection dates of Asial serotype FMDV

\begin{tabular}{|c|c|c|c|c|c|}
\hline Mark & Strain annotated in NCBI & Accession no. & Host annotated in NCBI & Region & Collection date \\
\hline Asial $\backslash 1$ & Vaccine IND $63 / 72$ & AY304994.1 & & India & 2003 \\
\hline Asiall2 & IND 334-00 & DQ989304.1 & Cattle & India & 2000 \\
\hline Asia1 3 & IND 151-94 & DQ989303.1 & Cattle & India & 1993 \\
\hline Asial 4 & IND $81-86$ & DQ989306.1 & Cattle & India & 1986 \\
\hline Asia1 5 & Asia1-2 & AY593796.1 & & Israel & 1963 \\
\hline Asial $\backslash 6$ & IND $397-97$ & DQ989308.1 & Cattle & India & 1997 \\
\hline Asial $\backslash 7$ & Asia1/YS/CHA/05 & GU931682.1 & Bovine & China & 2005 \\
\hline Asial $\backslash 8$ & ZB/CHA/58(att) & DQ533483.1 & Cattle & China & 1958 \\
\hline Asial $\ominus$ & Asia $1 / \mathrm{MOG} / 05$ & $\mathrm{EF} 614458.1$ & Cattle & Mongolia & 2005 \\
\hline Asial $\backslash 10$ & YNBS/58 & AY390432.1 & Cattle & China & 1958 \\
\hline Asial $\backslash 11$ & Asia1/Jiangsu/China/2005 & $\mathrm{EF} 149009.1$ & Bovine & China & 2005 \\
\hline Asial $\backslash 12$ & Asia1/Jiangsu/China/2005 & $\mathrm{EF} 149009.1$ & Bovine & China & 2005 \\
\hline Asia1 $\backslash 13$ & Asialleb4 iso4 & AY593799.1 & & Lebanon & 1983 \\
\hline Asial $\backslash 14$ & Asialleb-89 iso 89 & AY593798.1 & & Lebanon & 1983 \\
\hline Asial $\backslash 15$ & Asialleb83 iso28 & AY593800.1 & & Lebanon & 1983 \\
\hline Asia1 $\backslash 16$ & Asia1/WHN/CHA/06 & FJ906802.1 & Pig & China & 2002 \\
\hline Asial $\backslash 17$ & IND 116-90 & DQ989305.1 & Cattle & India & 1990 \\
\hline Asial $\backslash 18$ & asia1-1pak iso3 & AY593795.1 & Cattle & Pakistan & 1954 \\
\hline Asia1 $\backslash 19$ & Asia1/VN/QT03/2007 & GU125645.1 & Cattle & Viet Nam & 2007 \\
\hline Asial $\backslash 20$ & IND $82-96$ & DQ989309.1 & Cattle & India & 1996 \\
\hline Asial $\backslash 21$ & IND 247-92 & DQ989307.1 & Cattle & India & 1992 \\
\hline Asial $\backslash 22$ & IND 491/97; WBN 117/85 & AY687344.1 & & India & 1985 \\
\hline Asial $\backslash 23$ & IND 52-87 & DQ989313.1 & Buffalo & India & 1986 \\
\hline Asial $\backslash 24$ & IND 13-91 & DQ989312.1 & Sheep & India & 1990 \\
\hline Asial $\backslash 25$ & IND 148-01 & DQ989317.1 & Buffalo & India & 2000 \\
\hline Asial 126 & IND 47-93 & DQ989315.1 & Cattle & India & 1993 \\
\hline Asial $\backslash 27$ & Asia1/HNK/CHA/05 & $\mathrm{EF} 149010.1$ & Bovine & China & 2005 \\
\hline Asia1 $\backslash 28$ & IND 423-01 & DQ989319.1 & Cattle & India & 2001 \\
\hline Asial $\backslash 29$ & IND 321/01 & AY687333.1 & Cattle & India & 2001 \\
\hline$A \operatorname{sial} \backslash 30$ & IND 97-03 & DQ989323.1 & Cattle & India & 2002 \\
\hline Asia1 $\backslash 31$ & IND 37-02 & DQ989311.1 & Buffalo & India & 2002 \\
\hline Asial $\backslash 32$ & IND 139-02 & DQ989322.1 & Cattle & India & 2002 \\
\hline Asial $\backslash 34$ & IND 354-01 & DQ989314.1 & Cattle & India & 2001 \\
\hline Asia1 $\backslash 35$ & IND 438-01 & DQ989321.1 & Cattle & India & 2001 \\
\hline Asial $\backslash 36$ & IND 182-02 & DQ989320.1 & Cattle & India & 2002 \\
\hline Asial $\backslash 37$ & IND 101-99 & DQ989310.1 & Cattle & India & 1999 \\
\hline$A \operatorname{sia} 1 \backslash 38$ & MAY/9/99 & HQ632774.1 & Cattle & Malaysia & 1999 \\
\hline Asial $\backslash 41$ & IND $61-02$ & DQ989318.1 & Cattle & India & 2002 \\
\hline
\end{tabular}
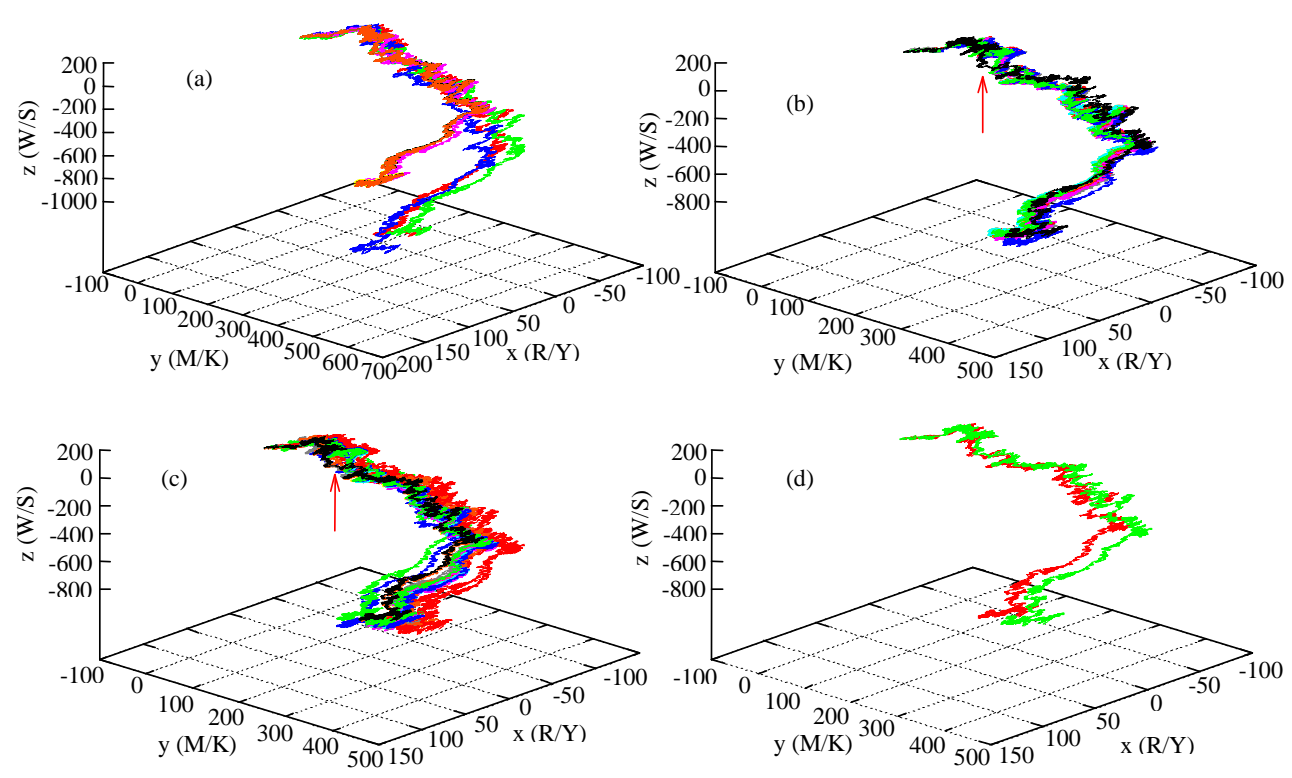

Fig. 2: a-d) The 3-dimensional curves of $O$ serotype FMDV isolated in swine 

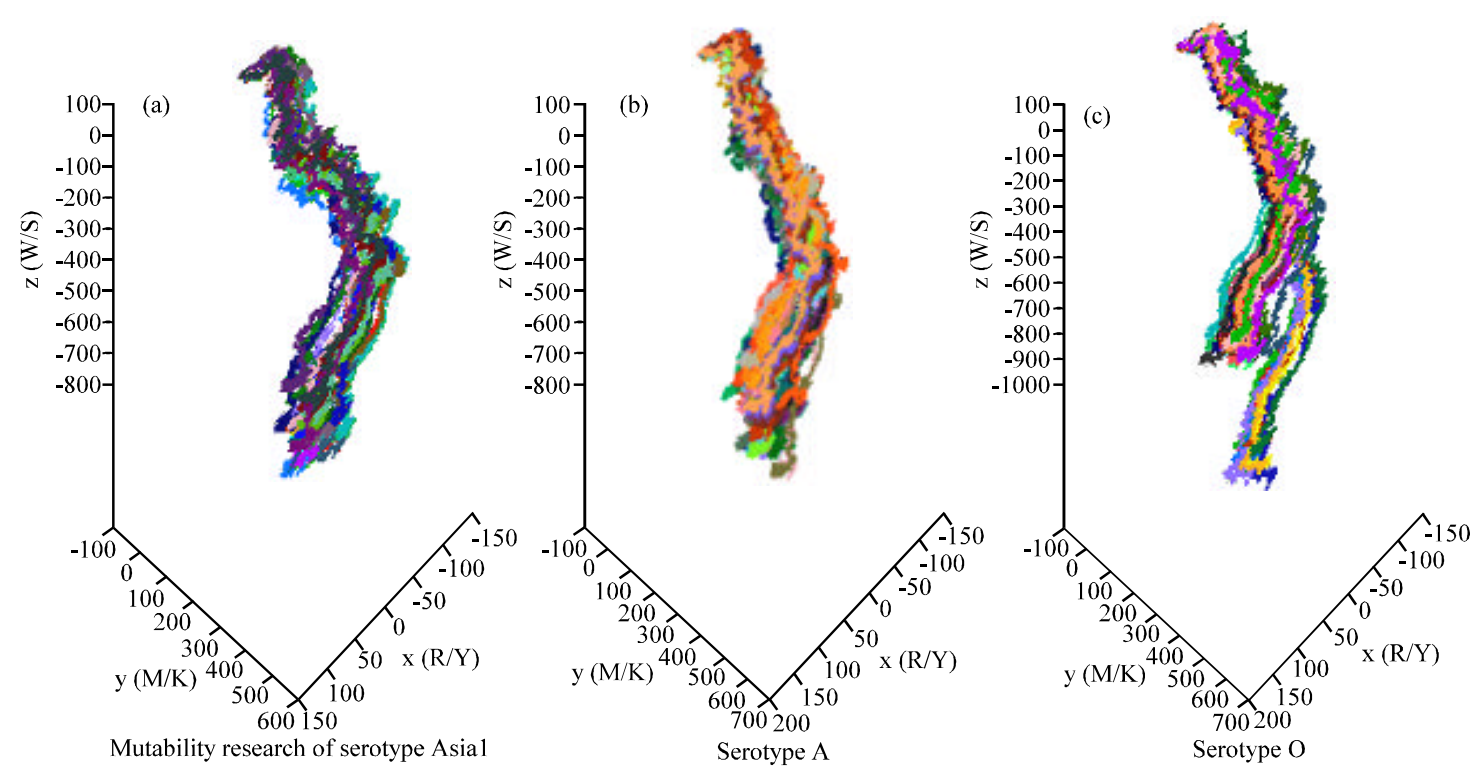

Fig. 3: a-c) The 3-dimensional curves of Asial, A and O serotypes FMDV. The O serotype FMDV is most variable

Table 3: The Z-curve plot marks, names, accession numbers, hosts as well as isolation regions and collection dates of A serotype FMDV shown in Fig. $3 \mathrm{~b}$

\begin{tabular}{|c|c|c|c|c|c|}
\hline Mark & Strain annotated in NCBI & Accession no. & Host annotated in NCBI & Region & Collection date \\
\hline a\1 & a1 0holland iso82 & AY593751.1 & & Netherlands & 1942 \\
\hline $\mathrm{a} / 2$ & A4 West Germany/ 72 & AY593779.1 & & Germany & 1972 \\
\hline a) 3 & a4 W Germany iso 42 & AY593777.1 & & Germany & 2004 \\
\hline a) 4 & a2spain iso 7 & AY593774.1 & & Spain & 1969 \\
\hline$a \backslash 6$ & albayern iso41 & AY593759.1 & & Germany & 1971 \\
\hline a)7 & a3mecklenburg iso 81 & AY593776.1 & & Germany & 1968 \\
\hline a\} 8 $&{\text { a12valle } 119 \text { iso20 }} &{\text { AY593752.1 }} &{ } &{\text { United Kingdom }} &{1932} \\
{\hline \text { a\} 1 0 } &{\text { a24cruzeiro iso } 71} &{\text { AY593768.1 }} &{ } &{\text { Brazil }} &{1955} \\
{\hline \text { a\} 1 1 } &{\text { a5westerwald iso } 73} &{\text { AY593781.1 }} &{ } &{\text { Germany }} &{1951} \\
{\hline \text { a\12 }} &{\text { A8 Parma aparma iso55 }} &{\text { AY593792.1 }} &{ } &{\text { Italy }} &{1962} \\
{\hline \text { a\13 }} &{\text { a5allier iso45 }} &{\text { AY593780.1 }} &{ } &{\text { France }} &{1960} \\
{\hline \text { a\14 }} &{\text { a20ussr iso } 10} &{\text { AY593760.1 }} &{ } &{\text { Russia }} &{1964} \\
{\hline \text { a\} 1 5 } &{\text { a14 spain iso39 }} &{\text { AY593754.1 }} &{ } &{\text { Spain }} &{1959} \\
{\hline \text { a\16 }} &{\text { a4spain iso62 }} &{\text { AY593778.1 }} &{ } &{\text { Spain }} &{2005} \\
{\hline \text { a\} 1 7 } &{\text { acanefa iso48 }} &{\text { AY593789.1 }} &{ } &{\text { Argentina }} &{1961} \\
{\hline \text { a\} 1 8 } &{\text { a } 25 \text { argentina iso } 38} &{\text { AY593769.1 }} &{ } &{\text { Argentina }} &{1959} \\
{\hline \mathrm{a} \backslash 19} &{\text { a17 Aguarulbos iso83 }} &{\text { AY593757.1 }} &{ } &{\text { Brazil }} &{1967} \\
{\hline \mathrm{a} \backslash 20} &{\text { a bage iso63 }} &{\text { AY593787.1 }} &{ } &{\text { Brazil }} &{1977} \\
{\hline \mathrm{a} \backslash 21} &{\text { a venceslau iso } 70} &{\text { AY593803.1 }} &{ } &{\text { Brazil }} &{1979} \\
{\hline \mathrm{a} \mid 22} &{\text { a18zulia iso40 }} &{\text { AY593758.1 }} &{ } &{\text { Venezuela }} &{1967} \\
{\hline \mathrm{a} \backslash 23} &{\text { a13brazil iso } 75} &{\text { AY593753.1 }} &{ } &{\text { Brazil }} &{1958} \\
{\hline \mathrm{a} \backslash 24} &{\text { a24 argentina iso } 9} &{\text { AY593767.1 }} &{ } &{\text { Argentina }} &{1965} \\
{\hline \text { a } 25} &{\text { a27columbia iso } 78} &{\text { AY593771.1 }} &{ } &{\text { Colombia }} &{1967} \\
{\hline \mathrm{a} / 26} &{\text { a16belem iso80 }} &{\text { AY593756.1 }} &{ } &{\text { Brazil }} &{1959} \\
{\hline \mathrm{a} \backslash 27} &{\text { a29peru iso37 }} &{\text { AY593773.1 }} &{ } &{\text { Peru }} &{1969} \\
{\hline \mathrm{a} \backslash 28} &{\text { a32ven iso36 }} &{\text { AY593775.1 }} &{ } &{\text { Venezuela }} &{1970} \\
{\hline \text { a } 29} &{\text { IND17/82 }} &{\text { HM854024.1 }} &{\text { Cattle }} &{\text { India }} &{1982} \\
{\hline \text { a) } 30} &{\text { a sabana iso68 }} &{\text { AY593794.1 }} &{ } &{\text { Colombia }} &{1985} \\
{\hline \text { a } 31} &{\text { a26arg iso } 74} &{\text { AY593770.1 }} &{ } &{\text { Argentina }} &{1966} \\
{\hline \text { a) } 32} &{\text { a brazil iso67 }} &{\text { AY593788.1 }} &{ } &{\text { Brazil }} &{1958} \\
{\hline \text { a) } 33} &{\text { aphilippines iso50 }} &{\text { AY593793.1 }} &{ } &{\text { Philippines }} &{1975} \\
{\hline \text { a)34 }} &{\text { aargp64 isol00 }} &{\text { AY593785.1 }} &{ } &{\text { Argentina }} &{2001} \\
{\hline \text { a) } 35} &{\text { aargp55 iso99 }} &{\text { AY593784.1 }} &{ } &{\text { Argentina }} &{2001} \\
{\hline \text { a\36 }} &{\text { a general lopez iso1 } 02} &{\text { AY593790.1 }} &{ } &{\text { Argentina }} &{2001} \\
{\hline \text { a) } 37} &{\text { aarg2001 iso93 }} &{\text { AY593783.1 }} &{ } &{\text { Argentina }} &{2001} \\
{\hline \text { a) } 38} &{\text { aarg Trenquelauquen iso103 }} &{\text { AY593786.1 }} &{ } &{\text { Argentina }} &{2001} \\
{\hline \text { a) } 39} &{\text { A uruguay } 2001 \text { iso98 }} &{\text { AY593802.1 }} &{ } &{\text { Uruguay }} &{2001} \\
\hline\end{array}$
\end{tabular}


J. Anim. Vet. Adv., 11 (15): 2630-2640, 2012

Table 3: Continue

\begin{tabular}{|c|c|c|c|c|c|}
\hline Mark & Strain annotated in NCBI & Accession no. & Host annotated in NCBI & Region & Collection date \\
\hline$\overline{\mathrm{a} \backslash 40}$ & A30 Uruguay/68 iso90 & AY593801.1 & & Uruguay & 1968 \\
\hline a)41 & IND17/77 & HM854022.1 & Cattle & India & 1977 \\
\hline a $\backslash 42$ & a28 Turkey iso 44 & AY593772.1 & & Turkey & 1972 \\
\hline a)43 & IND $109 / 2006$ & HQ832589.1 & Cattle & India & 2006 \\
\hline a)44 & a argentina 2000 iso 104 & AY593782.1 & & Argentina & 2000 \\
\hline a) 45 & IND 21/1990 & HQ832576.1 & & India & 1990 \\
\hline a) 46 & a23kenya iso8 & AY593766.1 & & Kenya & 1965 \\
\hline a 447 & a21kenya iso 77 & AY593761.1 & & Kenya & 1964 \\
\hline a) 48 & IND 110/1999 & HQ832577.1 & & India & 1999 \\
\hline a 49 & IND258/99 & HM854023.1 & Cattle & India & 1999 \\
\hline a\} 5 0 $&{\text { IND } 818 / 2003} &{\text { HQ832580.1 }} &{\text { Cattle }} &{\text { India }} &{2003} \\
{\hline \text { a } 51} &{\text { IND 281/2003 }} &{\text { HQ832579.1 }} &{\text { Buffalo }} &{\text { India }} &{2003} \\
{\hline \text { a } 52} &{\text { IND 43/2006 }} &{\text { HQ832586.1 }} &{ } &{\text { India }} &{2006} \\
{\hline \text { a } 53} &{\text { IND 245/2007 }} &{\text { HQ832590.1 }} &{\text { Cattle }} &{\text { India }} &{2007} \\
{\hline \mathrm{a} \backslash 54} &{\text { IND40/00 }} &{\text { HM854025.1 }} &{\text { Bovine }} &{\text { India }} &{1999} \\
{\hline \text { a) } 55} &{\text { IND } 447 / 2005} &{\text { HO832583.1 }} &{ } &{\text { India }} &{2005} \\
{\hline \mathrm{a} \backslash 57} &{\text { IND } 161 / 2003} &{\text { HQ832578.1 }} &{ } &{\text { India }} &{2003} \\
{\hline \text { a) } 58} &{\text { c1 ober iso } 88} &{\text { AY593805.1 }} &{ } &{\text { Germany }} &{1960} \\
{\hline \text { a } 59} &{\text { IND 26/2006 }} &{\text { HQ832585.1 }} &{ } &{\text { India }} &{2005} \\
{\hline \text { a) } 60} &{\text { IND81/00 }} &{\text { HM854021.1 }} &{\text { Buffalo }} &{\text { India }} &{2000} \\
{\hline \text { a) } 61} &{\text { a22iraq-95 iso95 }} &{\text { AY593762.1 }} &{ } &{\text { Iraq }} &{1964} \\
{\hline \text { a) } 62} &{\text { a22iraq64 iso86 }} &{\text { AY593763.1 }} &{ } &{\text { Iraq }} &{1964} \\
{\hline \text { a } 63} &{\text { IND 50/2006 }} &{\text { HQ832587.1 }} &{\text { Bullock }} &{\text { India }} &{2005} \\
{\hline \text { a) } 64} &{\text { a22iraq } 70 \text { iso92 }} &{\text { AY593764.1 }} &{ } &{\text { Iraq }} &{1970} \\
{\hline \text { a) } 65} &{\text { IND 88/2006 }} &{\text { HQ832588.1 }} &{\text { Cattle }} &{\text { India }} &{2005} \\
{\hline \text { a) } 66} &{\text { IND 64/2004 }} &{\text { HO832581.1 }} &{\text { Cattle }} &{\text { India }} &{2004} \\
{\hline \text { a) } 68} &{\text { IND 249/2004 }} &{\text { HQ832582.1 }} &{\text { Buffalo }} &{\text { India }} &{2004} \\
{\hline \text { a } 69} &{\text { a22turkey iso66 }} &{\text { AY593765.1 }} &{ } &{\text { Turkey }} &{1965} \\
{\hline \mathrm{a} \backslash 72} &{\text { IND } 437 / 2008} &{\text { HQ832591.1 }} &{\text { Cattle }} &{\text { India }} &{2008} \\
{\hline a \backslash 73} &{\text { IND } 17 / 2009} &{\text { HO832592.1 }} &{\text { Cattle }} &{\text { India }} &{2009} \\
{\hline \mathrm{a} \backslash 74} &{\text { a15thailand iso43 }} &{\text { AY593755.1 }} &{ } &{\text { Thailand }} &{1960} \\
{\hline \mathrm{a} \backslash 76} &{\text { IND 22/2006 }} &{\text { HQ832584.1 }} &{\text { Cattle }} &{\text { India }} &{2005} \\
{\hline a \backslash 77} &{\text { airan isol05 }} &{\text { AY593791.1 }} &{ } &{\text { Iran }} &{1998} \\
{\hline \text { a } 779} &{\mathrm{~A} / \mathrm{VN} / 03 / 2009} &{\text { GQ406249.1 }} &{\text { Cattle }} &{\text { Viet Nam }} &{2009} \\
{\hline \text { a) } 82} &{\mathrm{MAY} / 3 / 2007} &{\text { HQ632773.1 }} &{\text { Cattle }} &{\text { Malaysia }} &{2007} \\
{\hline \text { a) } 84} &{\mathrm{~A} / \mathrm{VN} / 16 / 2009} &{\text { GQ406251.1 }} &{\text { Pig }} &{\text { Viet Nam }} &{2009} \\
{\hline \text { ał } 85} &{\mathrm{~A} / \mathrm{VN} / 02 / 2009} &{\text { GQ406248.1 }} &{\text { Cattle }} &{\text { Viet Nam }} &{2009} \\
{\hline \text { a) } 86} &{\mathrm{~A} / \mathrm{VN} / 11 / 2009} &{\text { GQ406250.1 }} &{\text { Cattle }} &{\text { Viet Nam }} &{2009} \\
{\hline \text { ał } 88} &{\mathrm{~A} / \mathrm{VN} / 20 / 2009} &{\text { GQ406252.1 }} &{\text { Buffalo }} &{\text { Viet Nam }} &{2009} \\
{\hline \text { a)91 }} &{\text { A/VN/09/2009 }} &{\text { GQ406247.1 }} &{\text { Cattle }} &{\text { Viet Nam }} &{2009} \\
$\hline
\end{tabular}

Table 4: The Z-curve plot marks, names, accession numbers, hosts as well as isolation regions and collection dates of O serotype FMDV shown in Fig. 3c

\begin{tabular}{|c|c|c|c|c|c|}
\hline Mark & Strain annotated in NCBI & Accession no. & Host annotated in NCBI & Region & Collection date \\
\hline o\1 & Serotype="O" & AF308157.1 & Swine & Taiwan & 1997 \\
\hline $0 \backslash 2$ & Otaiwan97 iso106/112 & AY593835.1 & & Taiwan & 1997 \\
\hline$\circ 3$ & Openghu iso108 & AY593833.1 & & Taiwan & 1999 \\
\hline 016 & $\mathrm{O} / \mathrm{YM} / \mathrm{YN} / 2000$ & HQ412603.1 & Sus scrofa & China & 2000 \\
\hline 017 & $\mathrm{HKN} / 2002$ & AY317098.1 & & China & 2002 \\
\hline 018 & WFL & EF175732.1 & & China & \\
\hline ol9 & $\mathrm{lz}$ & DQ248888.1 & & China & \\
\hline $0 \backslash 10$ & $\mathrm{O} / \mathrm{ES} / 2001$ & AY686687.1 & & China & 2001 \\
\hline $0 \backslash 11$ & MAY/8/2005 & HQ632771.1 & Pig & Malaysia & 2005 \\
\hline $0 \backslash 12$ & $\mathrm{O} / \mathrm{HK} / 2001$ & EU400597.1 & & China & 2001 \\
\hline $0 \backslash 13$ & o5india iso34 & AY593828.1 & & India & 1962 \\
\hline $0 \backslash 14$ & Akesu/58 & AF511039.1 & Cattle epithelial blister & China & 1958 \\
\hline $0 \backslash 15$ & OGBF15 & DQ478936.1 & Cattle & China & \\
\hline \multirow[t]{2}{*}{$0 \backslash 16$} & OMIII artificially attenuated & & & & \\
\hline & from strain Akesu/ 58 & AY359854.1 & & China & 1958 \\
\hline $0 \backslash 17$ & OGBF15 derivative & DQ478937.1 & & China & \\
\hline $0 \backslash 18$ & o3venezuela iso15 & AY593827.1 & & Venezuela & 1971 \\
\hline $0 \backslash 19$ & olvalle iso64 & AY593825.1 & & Argentina & 1939 \\
\hline 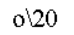 & o2brescia isol 7 & AY593826.1 & & Italy & 1947 \\
\hline $0 \backslash 21$ & Tibet/CHA/99 & AJ539138.1 & & China:Tibet & 1999 \\
\hline 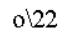 & olmanisa iso87 & AY593823.1 & & Turkey & 1969 \\
\hline $0 \backslash 23$ & olm11 iso57 & AY593822.1 & & & \\
\hline 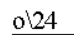 & China/1/99(Tibet) & AF506822.2 & & China:Tibet & \\
\hline
\end{tabular}


J. Anim. Vet. Adv., 11 (15): 2630-2640, 2012

Table 4: Continue

\begin{tabular}{|c|c|c|c|c|c|}
\hline Mark & Strain annotated in NCBI & Accession no. & Host annotated in NCBI & Region & Collection date \\
\hline 0425 & $\mathrm{TAW} / 2 / 99 \mathrm{BOV}$ & AJ539137.1 & & Taiwan & \\
\hline 0426 & $\mathrm{TAW} / 2 / 99 \mathrm{TC}$ & AJ539136.1 & & Taiwan & \\
\hline 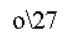 & $\mathrm{SAR} / 19 / 2000$ & AJ539140.1 & & South Africa & \\
\hline 0128 & UKG/4141/2001 & EF552689.1 & Sheep & United Kingdom & 2001 \\
\hline 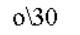 & MAY/1/2004 & HQ632770.1 & Cattle & Malaysia & 2004 \\
\hline ob31 & UKG/1734/2001 & FJ542368.1 & Cattle & United Kingdom & 2001 \\
\hline $0 \backslash 32$ & UKG/126/2001 & DQ404179.1 & Porcine & United Kingdom & 2001 \\
\hline $0 \backslash 33$ & $\mathrm{UKG} / 127 / 2001$ & DQ404178.1 & Porcine & United Kingdom & 2001 \\
\hline ob34 & UKG/128/2001 & DQ404177.1 & Porcine & United Kingdom & 2001 \\
\hline ob35 & UKG/150/2001 & DQ404176.1 & Bovine & United Kingdom & 2001 \\
\hline o\36 & FRA/1/2001 & AJ633821.1 & Bovine & France:Mayenne & 2001 \\
\hline 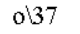 & UKG/35/2001 & AJ539141.1 & & United Kingdom & 2001 \\
\hline ob38 & UKG/7299/2001 & EF552692.1 & Sheep & United Kingdom & 2001 \\
\hline 0139 & $\mathrm{UKG} / 11 / 2001$ & DQ404180.1 & Porcine & United Kingdom & 2001 \\
\hline 0440 & $\mathrm{PAK} / 45 / 2008$ & GU384683.1 & Buffalo & Pakistan & 2008 \\
\hline $0 \backslash 41$ & $\mathrm{PAK} / 44 / 2008$ & GU384682.1 & Cattle & Pakistan & 2008 \\
\hline $0 \backslash 42$ & UKG/2085/2001 & FJ542370.1 & Cattle & United Kingdom & 2001 \\
\hline $0 \backslash 43$ & UKG/1558/2001 & FJ542367.1 & Cattle & United Kingdom & 2001 \\
\hline 0144 & UKG/7039/2001 & EF552690.1 & Cattle & United Kingdom & 2001 \\
\hline $0 \lcm{45}$ & UKG/3952/2001 & EF552688.1 & Sheep & United Kingdom & 2001 \\
\hline 0446 & UKG/9327/2001 & DQ404167.1 & Ovine & United Kingdom & 2001 \\
\hline 0447 & UKG/9788/2001 & DQ404166.1 & Bovine & United Kingdom & 2001 \\
\hline 048 & UKG/9964/2001 & DQ404165.1 & Bovine & United Kingdom & 2001 \\
\hline $0 \leq 50$ & UKG/8098/2001 & EU214601.1 & Sheep & United Kingdom & 2001 \\
\hline o\51 & UKG/4014/2001 & EF552693.1 & Cattle & United Kingdom & 2001 \\
\hline $0 \leq 52$ & UKG/220/2001 & DQ404173.1 & Bovine & United Kingdom & 2001 \\
\hline $0 \leq 53$ & $\mathrm{UKG} / 7038 / 2001$ & DQ404169.1 & Ovine & United Kingdom & 2001 \\
\hline $0 \backslash 54$ & UKG/9011/2001 & DQ404168.1 & Bovine & United Kingdom & 2001 \\
\hline $0 \backslash 55$ & $\mathrm{MAY} / \mathbf{3} / 2000$ & HQ632768.1 & Pig & Malaysia & 2000 \\
\hline 0156 & UKG/1450/2001 & FJ542366.1 & Sheep & United Kingdom & 2001 \\
\hline $0 \backslash 57$ & UKG/417/2001 & FJ542365.1 & Cattle & United Kingdom & 2001 \\
\hline 058 & UKG/5470/2001 & EF552696.1 & Cattle & United Kingdom & 2001 \\
\hline 0159 & UKG/4998/2001 & EF552694.1 & Cattle & United Kingdom & 2001 \\
\hline $0 \backslash 60$ & UKG/173/2001 & DQ404175.1 & Bovine & United Kingdom & 2001 \\
\hline $0 \backslash 61$ & UKG/438/2001 & DQ404174.1 & Ovine & Ireland & 2001 \\
\hline 0162 & UKG/4569/2001 & DQ404171.1 & Ovine & United Kingdom & 2001 \\
\hline $0 \backslash 63$ & UKG/7675/2001 & DQ404170.1 & Ovine & United Kingdom & 2001 \\
\hline $0 \backslash 64$ & UKG/11676/2001 & DQ404164.1 & Bovine & United Kingdom & 2001 \\
\hline $0 \backslash 65$ & O UK2001-FB & AY593832.1 & & United Kingdom & 2002 \\
\hline $0 \backslash 66$ & O UK2001-ED & AY593831.1 & & United Kingdom & 2002 \\
\hline $0 \backslash 67$ & $\mathrm{UKG} / 2526 / 2001$ & FJ542371.1 & Cattle & United Kingdom & 2001 \\
\hline $0 \backslash 68$ & UKG/2000/2001 & FJ542369.1 & Sheep & United Kingdom & 2001 \\
\hline 0169 & UKG/621/2001 & DQ404172.1 & Bovine & United Kingdom & 2001 \\
\hline$\circ>70$ & UKG/14476/2001 & DQ404162.1 & Ovine & United Kingdom & 2001 \\
\hline ol71 & UKG/14524/2001 & DQ404160.1 & Bovine & United Kingdom & 2001 \\
\hline $0 \backslash 72$ & O PanAsia uk2001 & AY593836.1 & Cattle & United Kingdom & 2001 \\
\hline 0173 & UKG/2640/2001 & FJ542372.1 & & United Kingdom & 2001 \\
\hline $0 \backslash 74$ & UKG/5681/2001 & EF552697.1 & Sheep & United Kingdom & 2001 \\
\hline $0 \backslash 75$ & UKG/9443/2001 & EF552695.1 & Sheep & United Kingdom & 2001 \\
\hline 0176 & UKG/9161/2001 & EF552691.1 & Cattle & United Kingdom & 2001 \\
\hline $0 \backslash 77$ & UKG/14391/2001 & DQ404161.1 & Bovine & United Kingdom & 2001 \\
\hline $0 \backslash 78$ & Israel 07-6380 & FJ175662.1 & Cattle & Israel & 2007 \\
\hline 0179 & o1 skr iso85 & AY593824.1 & & South Korea & 2000 \\
\hline o\80 & Israel 07-6389 & FJ175664.1 & Cattle & Israel & 2007 \\
\hline $0 \backslash 81$ & Israel 07-6378 & FJ175661.1 & Goat & Israel & 2007 \\
\hline $0 \backslash 82$ & UKG/14603/2001 & DQ404159.1 & Bovine & United Kingdom & 2001 \\
\hline o\83 & UKG/14339/2001 & DQ404163.1 & Bovine & United Kingdom & 2001 \\
\hline ol84 & $\mathrm{SKR} / 2000$ & AJ539139.1 & & South Korea & \\
\hline $0 \lcm{90}$ & UKG/15101/2001 & DQ404158.1 & Ovine & United Kingdom & 2001 \\
\hline 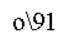 & Israel 07-6382 & FJ175663.1 & Cattle & Israel & 2005 \\
\hline 092 & Israel 07-6391 & FJ175665.1 & Cattle & Israel & 2007 \\
\hline ०७93 & orey-iran iso53 & AY593834.1 & & Iran & 1966 \\
\hline 0197 & o1 0phil76 iso76 & AY593812.1 & & Philippines & 1958 \\
\hline $0 \lcm{98}$ & o10phil54 iso54 & AY593811.1 & & Philippines & 1958 \\
\hline $0 \backslash 100$ & "O/SKR/14/02" & EF614457.1 & Cattle & South Korea & 2002 \\
\hline
\end{tabular}


Table 5: The Z-curve marks of those FMDV plotted in Fig. 1c, d, Fig. 2a-c, Fig. 4a and c. The strain names, accession numbers, hosts as well as isolation regions and collection dates are shown in Table 2-4

\begin{tabular}{|c|c|c|c|c|c|c|}
\hline Fig. 1c & Fig. 1d & Fig. 2a & Fig. $2 b$ & Fig. 2c & Fig. $4 \mathrm{a}$ & Fig. 4c \\
\hline ol32 & ol30 & o\11 & o\28 & o\14 & Asia1\2 & al29 \\
\hline ol33 & ol41 & ol6 & ol45 & o\15 & Asia1\3 & a)41 \\
\hline ol34 & o\14 & o\11 & ol50 & o\} 3 0 $&{\text { Asia1\4 }} &{\text { a)43 }} \\
{\hline \text { ol39 }} &{\text { o\} \backslash 1 5 } &{\text { ol55 }} &{\text { ol56 }} &{\text { ol31 }} &{\text { Asia1\6 }} &{\text { a)49 }} \\
{\hline \text { ol53 }} &{\text { ol78 }} &{\text { ol32 }} &{\text { ol68 }} &{\text { o\40 }} &{\text { Asia } 1 \backslash 17} &{\text { al50 }} \\
{\hline \text { o\61 }} &{\text { o\80 }} &{\text { ol33 }} &{\text { ol38 }} &{\text { o\41 }} &{\text { Asia } 1 \backslash 20} &{\text { al51 }} \\
{\hline \text { o\62 }} &{\text { o\100 }} &{\text { ol34 }} &{\text { o\46 }} &{\text { o\42 }} &{\text { Asia } 1 \backslash 23} &{\text { al53 }} \\
{\hline \text { o\63 }} &{\text { o\31 }} &{\text { ol39 }} &{\text { o\53 }} &{\text { ol43 }} &{\text { Asia } 1 \backslash 24} &{\text { al54 }} \\
{\hline \text { o\47 }} &{\text { o\42 }} &{ } &{\text { o\61 }} &{\text { ol44 }} &{\text { Asia } 1 \backslash 25} &{\text { a)60 }} \\
{\hline \text { o\48 }} &{\text { o\43 }} &{ } &{\text { o\62 }} &{\text { ol47 }} &{\text { Asia } 1 \backslash 26} &{\text { a)61 }} \\
{\hline \text { ol52 }} &{\text { ol89 }} &{ } &{\text { o\63 }} &{\text { o\48 }} &{\text { Asia } 1 \backslash 28} &{\text { a)63 }} \\
{\hline \multirow[t]{10}{*}{\text { ol53 }}\text { ol53 }} &{\text { o\91 }} &{ } &{\text { o\70 }} &{\text { o\51 }} &{\text { Asia } 1 \backslash 30} &{\text { a)65 }} \\
{\hline} &{ } &{ } &{\text { o\90 }} &{\text { o\52 }} &{\text { Asia } 1 \backslash 31} &{\text { a)66 }} \\
{\hline} &{ } &{ } &{\text { ol81 }} &{\text { ol53 }} &{\text { Asia } 1 \backslash 32} &{\text { al72 }} \\
{\hline} &{ } &{ } &{ } &{\text { ol57 }} &{\text { Asia } 1 \backslash 34} &{\text { al73 }} \\
{\hline} &{ } &{ } &{ } &{\text { ol58 }} &{\text { Asia } 1 \backslash 35} &{\text { al76 }} \\
{\hline} &{ } &{ } &{ } &{\text { ol60 }} &{\text { Asia } 1 \backslash 36} &{ } \\
{\hline} &{ } &{ } &{ } &{\text { ol78 }} &{\text { Asia } 1 \backslash 37} &{ } \\
{\hline} &{ } &{ } &{ } &{\text { o\80 }} &{\text { Asia1\41 }} &{ } \\
{\hline} &{ } &{ } &{ } &{\text { ol89 }} &{ } &{ } \\
{\hline} &{ } &{ } &{ } &{o \backslash 100} &{ } &{ } \\
\hline\end{array}$
\end{tabular}
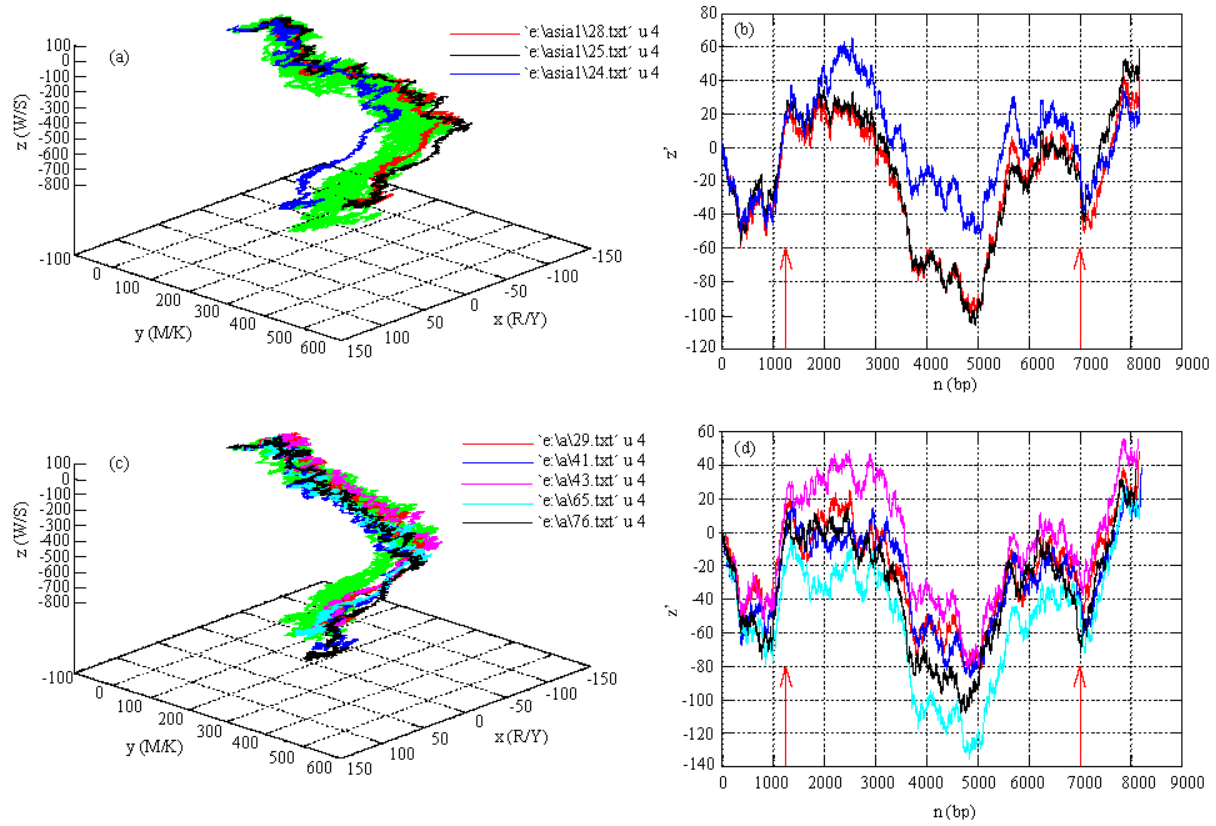

Fig. 4: a-d) The 3-dimensional curve of Asia1 serotype FMDV occurred in India before 2002

What's more, the $\mathrm{O}$ serotype FMDV are distinctly divided into two clusters according to the 3-dimensional curves (Fig. 3c).

Study on the Asia1 and A serotype FMDV which occurred in India: Statistics in Table 4 and 5 show that the mainly epidemic FMDV in India before 2002 were Asia1 serotype whereas it changed to A serotype after 2002. They are extracted out and conducted Z-curve analysis (Table 3-5). The results illustrate that the Asial serotype FMDV strains which were epidemic in India before 2002 varied too much (Fig. 4a, b). The A serotype FMDV strains occurred in India after 2002 are more stable and have a closer phylogenetic relationships compared with the Asia1 strains which were prevalent before 2002 (Fig. $4 \mathrm{c}, \mathrm{d}$ ). The variation regions exactly locate in the protein coding areas and the non-coding region is very conservative (Fig. $4 \mathrm{~b}, \mathrm{~d}$ ). Figure $4 \mathrm{c}$ shows the 3-dimensional curves of A serotype FMDV occurred in India after 2002. As can be observed, these curves can be divided into a similarity cluster and a dispersity cluster. Researchers plot the Z-curves of these dispersity clusters, 
respectively in Fig. 4b, d. Results indicate that the A serotype strains occurred in India after 2002 are more stable and have a closer phylogenetic relationships compared with the Asial strains which were prevalent before 2002. What's more, the variation regions exactly locate in the protein coding areas.

FMDV has been considered as the most economically severe animal virus with a remarkable degree of antigenic diversity (Damien and Fares, 2006). It has a high mutation rate and spontaneous mutants which may be readily isolated in the laboratory (McCahon et al., 1977). The biological significance of the rapid evolution of RNA viruses have become increasingly obvious in systems such as infections by influenza virus, poliovirus and vesicular stomatitis virus (Holland et al., 1982; Holland, 1984). As is known to all, the genetic diversity of FMDV is a consequence of the high mutation rate due to the error-prone RNA poly-merase lacking proof reading activity. It has been recognized that FMDV is undergoing a rapid evolution in the field situation (Kumar et al., 2004). FMDV evolution is strongly influenced by high mutation rates and a quasispecies dynamics (Domingo et al., 2003). As argued in this study, geographic location plays a key role in the evolution of FMDV. The variability in FMDV (RNA) caused by high errors rates during their replication seems to be crucial for the survival of virus populations when the environmental conditions are altered. Damien and Fares (2006) suggested that serotypes with wide geographical distribution have accumulated compensatory mutations as a strategy to ameliorate the effect of slightly deleterious mutations fixed by genetic drift. Also, we find that host play an important role in the variation of FMDV. Researchers have proved that selection exerted by host antibody also plays a major role in the rapid evolution of FMDV Asial as observed in other serotypes. What's more, researchers find that compared with host, geographic location have a grater influence on FMDV mutation.

In this study, researchers find that the most variable regions of FMDV lie in protein coding areas and the non-coding region is very conservative. Insights into viral RNA sequence conservation and variability in nature will likely impact the understanding of FMDV infections, host range and transmission (Carrillo et al., 2005). As can be observed in Z-curve, researchers know that Z-curve can also analyze the structural proteins and non-structural proteins coding genes. Researchers know that VP1 is a structural protein of FMDV. It has been long recognized that the VP1 gene differs in about $30-50 \%$ between serotypes (Knowles and Samuel, 2003). It is recognized that non-structural proteins, crucial roles for viral propagation are more conserved than the structural proteins. Most mutations or deletions in the non-structural proteins could be detrimental to viral replication and protein processing. Converged mutations in structural proteins may help FMDV to evade immune response mounted by the host while maintaining the functional capsid. Consequently, the gene sequences of the structural proteins could mutate with a higher frequency for retaining evolutional advantages (Feng et al., 2004).

In addition, researchers find $Z$-curve is also helpful to study the molecular epidemiology of FMDV. The use of molecular epidemiology is an important tool in understanding and consequently controlling FMDV (Joern, 2009). As mentioned before, Israel 07-6378 (goat) and PAK/45/2008 (buffalo) are very similar while they are different from others. So, it indicates that there is a variation trend in buffalo and goat of O serotype FMDV. If we take the collection time and isolation region of them into consideration, we should ponder whether or not there is a relationship between their occurrence. If there is, how does the virus transmit from Israel to Pakistan, how does it conquer the species diversity from goat to buffalo, etc. Researchers also analyzed the mainly FMDV prevalent in India, researchers find that the A serotype FMDV strains which occurred in India after 2002 are more stable and have a closer phylogenetic relationship than the Asial serotype FMDV strains which were epidemic before 2002 . All these enlighten us that Z-curve is helpful to study the molecular epidemiology of FMDV. An increased understanding of how FMDV strains move between geographic regions will play a pivotal role in the development of future disease control strategies (Knowles and Samuel, 2003).

In this study, researchers can see that Z-curve also show the ability of homology analysis. According to Fig. $3 c$, the $O$ serotype FMDV are distinctly divided into two clusters. What's more, researchers find that all serotypes FMDV have a close consanguinity but each has its own characteristic by contrast, the three South Africa FMDV have a much closer affinity. Researchers can distinguish the kinship from the overlap extent and the similarity of 3-dimensional Z-curves and Z-curves. However, this kind of means is only appropriate for analyzing a small quantity of data. We should explore more about $Z$-curve if we want to analyze large amount of data. Such as measuring the differences among the 3-dimensional Z-curves.

Zheng et al. (2005) use the geometric center and the eigenvectors to describe evolutionarily of FMDV. They constructed a phylogenetic tree of the analyzed FMDV and found that it is consistent with those of previous analyses (Zheng et al., 2005). 


\section{CONCLUSION}

In general, Z-curve Method shows a widely application prospect in the areas of life sciences. Such as gene sequence, molecular epidemiology, homology, evolution, phylogenetic relationships and genetic diversity analysis. In addition, the Z-curve also can be used to recognize protein coding genes. Z-curve_E, a new self-training system derived from $Z$-curve has been used to recognize protein coding genes (Guo and Zhang, 2006). Currently, the Z-curve Method is still in its premature stage. Since, the more information is extracted from the Z-curve, the more accurate result can be gained, researchers are expected to explore more about the Z-curve. Researchers look forward to exploit some novel and more effective algorithms to extract information contained in the Z-curves.

\section{ACKNOWLEDGEMENTS}

This study was financially supported by the National High Technology Research and Development Program of China (863 Program) (No. 2011AA10A211) and Ministry of Agriculture' Special Funds for Scientific Research on Public Causes (No. 201203039).

\section{REFERENCES}

Alexandersen, S. and N. Mowat, 2005. Foot-and-mouth disease: Host range and pathogenesis. Curr. Top. Microbiol. Immunol., 288: 9-42.

Barasa, M., A. Catley, D. Machuchu, H. Laqua, E. Puot, D.T. Kot and D. Ikiror, 2008. Foot-and-mouth disease vaccination in South Sudan: Bene?t-cost analysis and livelihoods impact. Transbound. Emerg. Dis., 55: 339-351.

Carrillo, C., E.R. Tulman, G. Delhon, Z. Lu and A. Carreno et al., 2005. Comparative genomics of foot-and-mouth disease virus. J. Virol., 79: 6487-6504.

Chu, J.H., S.T. Weiss, V.J. Carey and B.A. Raby, 2009. A graphical model approach for inferring large-scale networks integrating gene expression and genetic polymorphism. BMC. Syst. Biol. Vol. 33. 10.1186/1752-0509-3-55

Cottam, E.M., J. Wadsworth, A.E. Shaw, R.J. Rowlands and L. Goatley et al., 2008. Transmission pathways of foot-and-mouth disease virus in the United Kingdom in 2007. PLoS. Pathog. Vol. 4.

Damien, C.T. and M.A. Fares, 2006. Unravelling selection shifts among foot-and-mouth disease virus (FMDV) serotypes. Evol. Bioinform. Online, 2: 211-225.
Davies, G., 2002. Foot and mouth disease. Res. Vet. Sci., 73: 195-199.

Domingo, E., C. Escarmis, E. Baranowski, C.M. RuizJarabo, E. Carrillo, I.J. Nunez and F. Sobrino, 2003. Evolution of foot-and-mouth disease virus. Virus Res., 91: 47-63.

Feng, Q., H. Yu, Y. Liu, C. He and J. Hu et al., 2004. Genome comparison of a novel foot-and-mouth disease virus with other FMDV strains. Biochem. Biophys. Res. Commun., 323: 254-263.

Giacomo, F., M.M. Francesco, C. Davide and H. Muller, 2007. Graph-based identification of cancer signaling pathways from published gene expression signatures using PubLiME. Nucleic Acids Res., 35: 2343-2355.

Gibbs, P., 2003. The foot-and-mouth disease epidemic of 2001 in the UK: implications for the USA and the J. Vet. Med. Educ., 30: 121-132.

Guo, F.B. and C.T. Zhang, 2006. ZCURVE_V: A new selftraining system for recognizing protein-coding genes in viral and phage genomes. BMC Bioinformatics, Vol. 7.

Guo, F.B. and X.J. Yu, 2007. Separate base usages of genes located on the leading and lagging strands in Chlamydia muridarum revealed by the $Z$ curve method. BMC Genomics, 8: 1471-2164.

Guo, F.B., H.Y. Ou and C.T. Zhang, 2003. ZCURVE: A new system for recognizing protein-coding genes in bacterial and archaeal genomes. Nucleic Acids Res., 31: 1780-1789.

Holland, J.J., 1984. Continuum of Change in RNA Virus Genomes. In: Concepts in Viral Pathogenesis, Notkins, A.L. and M.B.A. Oldstones (Eds.). Spinger Wien, New York, pp: 137-143.

Holland, J.J., J.C. De La Torre and D.A. Steinhauer, 1982. RNA virus populations as quasispecies. Curr. Top. Microbiol. Immunol., 176: 1-20.

Joern, K., 2009. Understanding the molecular epidemiology of foot-and-mouth-disease virus. Infect. Genet. Evol., 9: 153-161.

Knowles, N.J. and A.R. Samuel, 2003. Molecular epidemiology of foot and mouth disease virus. Virus. Res., 91: 65-80.

Kumar, R.M., A. Sanyal, D. Hemadri, C. Tosh, J.K. Mohapatra and S.K. Bandyopadhyay, 2004. Characterization of foot-and-mouth disease serotype asial viruses grown in the presence of polyclonal antisera in serology and nucleotide sequence analysis. Arch. Virol., 149: 1801-1814.

Li, X., C. Panea., C.H. Wiggins, V. Reinke and C. Leslie, 2010. Learning graph-mer motifs that predict gene expression trajectories in development. PLoS Comput. Biol. Vol. 6. 10.1371/journal.pcbi.1000761 
McCahon, D., W.R. Slade, R.A.J. Priston and J.R. Lake, 1977. An extended genetic recombination map of foot-and-mouth disease virus. J. Gen. Virol., 35: $555-565$.

Mort, M., I. Convery, J. Baxter and C. Bailey, 2005. Psychosocial effects of the 2001 UK foot-and-mouth disease epidemic in a rural population: Qualitative diary based study. BMJ Vol. 331.

Ou, H.Y., F.B. Guo and C.T. Zhang, 2003. Analysis of nucleotide distribution in the genome of streptomyces coelicolor A3(2) using the $Z$ curve method. FEEBS. Lett., 540: 188-194.

Paprocka, G., 2006. Foot-and-mouth disease virus and its molecular structure. Med. Weter., 62: 753-756.

Perry, B.D. and K.M. Rich, 2007. Poverty impacts of footand-mouth disease and the poverty reduction implications of its control. Vet. Rec., 160: 238-241.

Rodriguez, L.L. and M.J. Grubman, 2009. Foot-and-mouth disease virus vaccines. Vaccine, 27: 90-94.

Rufael, T., A. Catley, A. Bogale, M. Sahle and Y. Shiferaw, 2008. Foot-and-mouth disease in the Borana pastoral system, southern Ethiopia and implications for livelihoods and international trade. Trop. Anim. Health. Pro., 40: 29-38.

Schumann, K.R., N.J. Knowles, P.R. Davies, R.J. Midgley and J.F. Valarcher et al., 2008. Genetic characterization and molecular epidemiology of footand-mouth disease viruses isolated from Afghanistan in 2003-2005. Virus Genes, 36: 401-413.

Sobrino, F., M. Saiz, M.A. Jimenez-Clavero, J.I. Nunez, M.F. Rosas, E. Baranowski and V. Ley, 2001. Footand-mouth disease virus: A long known virus, but a current threat. Vet. Res., 32: 1-30.
Sumption, K., M. Rweyemamu and W. Wint, 2008. Incidence and distribution of foot-and-mouth disease in Asia, Africa and South America, combining expert opinion, official disease information and livestock populations to assist risk assessment. Transbound. Emerg. Dis., 55: 5-13.

Thompson, D., P. Muriel, D. Russell, P. Osborne, A. Bromley, M. Rowland, T.S. Creigh, and C. Brown, 2002. Economic costs of the foot- and-mouth disease outbreak in the United Kingdom in 2001. Rev. Sci. Tech., 21: 675-687.

Wen, S.Y. and C.T. Zhang, 2003. Identification of isochore boundaries in the human genome using the technique of wavelet multiresolution analysis. Biochem. Biophys. Res. Commun., 311: 215-222.

Zhang, C.T. and F. Zhang, 2003a. An isochore map of the human genome based on the $Z$ curve method. Gene., 317: $127-135$.

Zhang, C.T. and J. Wang, 2000. Recognition of protein coding genes in the yeast genome at better than $95 \%$ accuracy based on the $Z$ curve. Nucleic Acids Res., 28: 2804-2814.

Zhang, R. and C.T. Zhang, 2003b. Identification of genomic islands in the genome of Bacillus cereus by comparative analysis with Bacillus anthracis. Physiol. Genomics., 16: 19-23.

Zhang, R. and C.T. Zhang, 2005. Identification of replication origins in archaeal genomes based on the Z-curve method. Archaea, 1: 335-346.

Zheng, W.X., L.L. Chen, H.Y. Ou, F. Gao and C.T. Zhang, 2005. Coronavirus phylogeny based on a geometric approach. Mol. Phylogenet. Evol., 36: 224-232. 